\title{
UNILATERAL STIFFNESS INTERVENTIONS AUGMENT VERTICAL STIFFNESS AND CHANGE OF DIRECTION SPEED
}

\section{RUNNING HEAD}

Stiffness interventions on CODS

\section{KEYWORDS}

Plyometrics, Pre-conditioning, Warm-up, Post-activation potentiation, Agility

\section{ACKNOWLEDGEMENTS}

No benefits in any form have been or will be received from any commercial party/grant body related directly or indirectly in relation to this manuscript. The results of the present study do not constitute the endorsement of any product by the authors or by the NSCA. 


\section{ABSTRACT}

It has previously been shown that pre-conditioning interventions can augment change of direction speed (CODS). However, the mechanistic nature of these augmentations has not been well considered. The current study sought to determine the effects of pre-conditioning interventions designed to augment vertical stiffness on CODS. Following familiarization, ten healthy males (age: $22 \pm 2$ years; height: $1.78 \pm 0.05 \mathrm{~m}$; body mass: $75.1 \pm 8.7 \mathrm{~kg}$ ) performed three different stiffness interventions in a randomized and counterbalanced order. The interventions were: a) bilateral-focused, b) unilateral-focused, and c) a control of CODS test practice. Vertical stiffness and joint stiffness was determined pre- and post-intervention using a single leg drop jump task. CODS test performance was assessed post-intervention using a double $90^{\circ}$ cutting task. Performances following the unilateral intervention were significantly faster than control $(1.7 \% ; P=0.011 ; d=-1.08)$, but not significantly faster than the bilateral intervention ( $1.0 \%$ faster; $P=0.14 ; d=-0.59)$. Versus control, vertical stiffness was $14 \%$ greater $(P=0.049 ; d=0.39)$ following the unilateral intervention and $11 \%$ greater $(P=0.019 ; d=0.31)$ following the bilateral intervention; there was no difference between unilateral and bilateral interventions $(P=0.94 ; d=-0.08)$. The findings of the current study suggest that unilateral pre-conditioning interventions designed to augment vertical stiffness improve CODS within this experimental cohort. 


\section{INTRODUCTION}

The ability to quickly and effectively change direction underpins performance in a wide range of sports. For example, change of direction speed (CODS) has been linked to performance in soccer (30), rugby league (25) and field hockey (14). Interventions designed to improve CODS are therefore likely to carry a beneficial effect to performance. Acute pre-conditioning interventions employing heavy resistance exercise (41) and loaded ballistic exercise (i.e. weight vest loaded warm-up) $(22,27)$ have been demonstrated to favourably affect CODS although the reasons behind these performance enhancements are yet to be elucidated.

Young, et al. (39) outlined three physical factors which may underpin CODS: strength (allied to maximal force production), power (allied to rate of force development) and reactive strength (allied to parameters of stiffness). This current study acknowledges the contribution of these qualities in the proposition of a slightly modified deterministic model of CODS as shown in Figure 1.

\section{${ }^{* * *}$ Figure 1 Near Here ${ }^{* * *}$}

Explanations of the performance benefits associated with pre-conditioning interventions have previously focused on how physiological and neural mechanisms contribute to enhancements in maximal force and rate of force development by way of post-activation potentiation (see Maloney, et al. (21) for a review). A growing body of evidence is now showing that pre-conditioning interventions may also augment stiffness $(2,9,26)$, highlighted by the inclusion of stiffness in a deterministic model of jump potentiation (34). 
Vertical stiffness describes the vertical displacement of the centre of mass in response to vertical ground reaction force during sagittal plane movement (17) and seeks to approximate the deformation of the leg-spring at instants of ground contact (5). Heavy resistance exercise has been shown to augment vertical stiffness in studies by Comyns, et al. (9) and Moir, et al. (26), the investigators noting increases of $10.9 \%(P$ $<0.05)$ and $16 \%(d: 0.52 ; P=0.013)$ respectively. A weight vest loaded warm-up has also been demonstrated to augment vertical stiffness by $20 \%$ ( $d: 0.76 ; 90 \%$ confidence intervals: 4\%) during a plyometric jumping task (2).

Acute augmentations in stiffness may be hypothesized to improve CODS performance. It has previously been demonstrated that vertical stiffness derived from a single leg drop jump explained $50 \%$ of the variance in CODS performance $\left(R^{2}=\right.$ $0.50, P=0.001)$ in a recreationally trained cohort (20). In addition, faster performers during the CODS task exhibited greater vertical stiffness $(d: 1.76 ; P=0.003) \quad(20)$. Greater stiffness would be expected to facilitate efficient transmission of the generated impulse and minimize the required ground contact time for the direction change to be executed (4). Previous research has reported that faster performers exhibit shorter ground contact times than slower performers in CODS tasks $(20,23,31,33)$. This is perhaps indicative of greater vertical stiffness given the relationship between vertical stiffness and ground contact time observed by Arampatzis, et al. (1), increases in stiffness were associated with reduced contact times.

The potential importance of vertical stiffness to CODS may also be evidenced given the relationship between reactive strength and CODS performance. Young, et al. (39) reported that reactive strength index was the physical variable which demonstrated the strongest relationship with CODS test time $(r=-0.54 ; P<0.05)$. Similar relationships have since been observed by Young, et al. (40) $(r=-0.65 ; P=0.001)$ 
and Delaney, et al. (10) (dominant limb: $R=-0.44 ; P<0.05$, non-dominant limb: $r=-$ $0.45 ; P<0.05)$. Reactive strength index is a function of the flight time or jump height divided by ground contact time recorded during a drop jump (28). The likely reduction in ground contact time associated with greater vertical stiffness (1) would therefore increase reactive strength index if the same jump height can be maintained. The deterministic model of potentiation detailed by Suchomel, et al. (34) emphasized such a reliance of reactive strength on stiffness.

Plyometric activities have been shown to augment performance in ballistic performance tasks including jumps $(3,7,16)$ and sprints $(6,18)$. The majority of investigations have utilized drop jumps as the potentiating stimulus $(6,7,18)$. Other investigations have employed conditioning hops (often termed pogo hops) $(3,16)$, alternate-leg bounding (36) and a mixed-activity (pogo hops, hurdle jumps and drop jumps) stimulus (35). To date, the effect of a plyometric pre-conditioning intervention on CODS has not been investigated. Given the positive results seen in the aforementioned investigations, it may appear likely that CODS would be similarly augmented.

Previous investigations have not sought to determine a mechanistic basis for the acute enhancement of CODS. Given the potential importance of stiffness in maximising CODS it is possible that the performance improvements observed following preconditioning interventions are related to augmentations in stiffness, however, such propositions must be examined directly. The aim of the current study was therefore to establish if acute exercise interventions designed to augment vertical stiffness influenced CODS. It was hypothesized that both bilateral and unilateral stiffness interventions would significantly improve CODS test performance versus a control strategy of additional CODS practice. 


\section{METHOD}

\section{EXPERIMENTAL APPROACH TO THE PROBLEM}

The current study was a repeated measures experiment designed to compare the effects of different pre-conditioning interventions on stiffness, asymmetries and CODS. Following a familiarization session, participants performed three different 'stiffness' interventions in a randomized and counterbalanced order. The three interventions were a) bilateral-focused (BILATERAL), b) unilateral-focused (UNILATERAL), and $\mathrm{c}$ ) a control of CODS test practice (CONTROL). Vertical stiffness was determined pre- and post-intervention whilst CODS test performance was assessed post-intervention.

\section{SUBJECTS}

Fourteen healthy males volunteered to participate in the study. Ten participants completed all three experimental trials (age: $22 \pm 2$ years; height: $1.78 \pm 0.05 \mathrm{~m}$; body mass: $75.1 \pm 8.7 \mathrm{~kg}$ ). A minimum sample size of nine participants was determined from a priori power analysis (G*Power 3.1, Heinrich-Heine-Universität, Düsseldorf, Germany) based upon an estimated effect size (d) of 0.6 and a power of 0.8 . Participants were recreationally active (undertaking $\geq 2.5$ hours of physical activity per week), reported no previous (within the last 12 months) or present lower limb injury and provided informed consent to participate in the study. Full ethical approval was granted by the relevant institutional review board. 


\section{PROCEDURES}

A single familiarization session was performed seven days prior to the experimental trial. During the session, participants were familiarized with the testing procedures and warm-up exercises.

${ }^{* \star *}$ Figure 2 Near Here ***

An outline of the experimental trials is shown in Figure 2. All trials were conducted at the same time of day (09:30 -12:00) for each participant, to alleviate the effects of circadian rhythms. The testing laboratory was controlled at an ambient temperature of $25^{\circ} \mathrm{C}$. Participants were instructed to prepare for testing as they would for training. The execution of each experimental trial was monitored by a NSCA Certified Strength and Conditioning Specialist to ensure for consistency of technique.

\section{WARM-UP}

Participants completed 5 minutes of cycle ergometry at a self-determined power output $(135 \pm 22 \mathrm{~W})$. During the familiarization session, participants were instructed to find a cadence and loading which allowed them to achieve a rating of perceived exertion of 5-7 (0-10 scale), this cadence and loading combination was then employed during the experimental trials. Following the cycle ergometry warm-up, participants performed a series of mobility exercises (Figure 2). These comprised of the following exercises: inchworm, quadruped thoracic rotation, push up to ' $\mathrm{T}$ ', supine glute bridge with abduction, mountain climber, squat to stand, single-leg stiff-legged deadlift to reverse lunge.

STIFFNESS INTERVENTIONS

${ }^{* * *}$ Table 1 Near Here ${ }^{* * *}$ 
Participants completed three experimental trials associated with the three different stiffness interventions outlined in Table 1; trials were separated by no less than six and no more than fourteen days. For the unilateral exercises, the number of prescribed repetitions was performed on both legs. For the bilateral and unilateral exercises, sets and exercises were separated by 60 seconds (29), in the unilateral intervention there was no recovery between limbs for any of the exercises. Bilateral drop jumps were performed from a greater height than unilateral drop jumps to partially offset discrepancies in the vertical ground reaction forces experienced at ground contact. For the control intervention, participants performed circuits of the CODS test. CODS practice was chosen as the control intervention as this would be more representative of a 'typical' warm-up strategy which would attempt to replicate the types of subsequent activity to be undertaken (24). Circuits of the CODS test were performed alternating between clockwise and counter-clockwise directions, each separated by 60 seconds. Participants were instructed to perform the first circuit at $50 \%$ intensity and the subsequent four with maximal effort.

\section{STIFFNESS ASSESSMENT}

Vertical and joint stiffness of the left and right limbs were assessed before and after the stiffness intervention (Figure 2) using a single leg drop jump protocol. This protocol has been described in detail in a previous manuscript (19). Participants performed two drop jumps, without footwear, for each limb at each time point. Drop jumps were performed from a height of $0.18 \mathrm{~m}$ onto a force plate system (Kistler 9281, Kistler Instruments, Winterthur, Switzerland) and were recorded in the sagittal plane using a high-speed video camera (Quintic High-Speed LIVE USB 2, Quintic Consultancy Ltd., Coventry, United Kingdom) at a frame-rate of $100 \mathrm{~Hz}$. Recordings were automatically digitized using manufacturer provided software (Quintic Biomechanics v21, Quintic 
Consultancy Ltd., Coventry, United Kingdom). Data were filtered using a Butterworth fourth-order zero-lag filter (cut-off frequency $20 \mathrm{~Hz}$ ).

Inverse dynamics was used to express acceleration, velocity and centre of mass displacement; this was determined from the vertical force trace. Net muscle moments were determined using a rigid linked segment model, anthropomorphic data and an inverse dynamics analysis using the procedures outlined by Winter (38); the linked segment model was created using Dempster's body segment parameter data (11).

Vertical stiffness was calculated as the ratio of peak vertical ground reaction force $(\mathrm{kN})$ relative to peak centre of mass displacement $(\mathrm{m})$ during the initial ground contact phase (12); this was reported relative to body mass and was averaged over the two drop jumps. Torsional stiffness of the ankle and knee joints were calculated as the ratio of the change in net muscle moment $(\mathrm{N})$ to joint angular displacement (rad) between the initial ground contact phase and instant of peak angular displacement (12); these were also averaged over the two jumps. Data for hip stiffness were excluded as the phase shift for the moment displacement curve of the hip was $>10 \%$, previously specified as exclusion criteria $(12,15,19)$.

\section{CHANGE OF DIRECTION SPEED TESTING}

\section{${ }^{* * *}$ Figure 3 Near Here ***}

CODS performance was assessed following each of the stiffness interventions (Figure 2) using the double-cut test shown in Figure 3. Participants were required to perform two $90^{\circ}$ cuts in the same direction (clockwise for the left leg trials or counter-clockwise for the right leg trials) during each trial and were instructed to complete the task as quickly as possible. Performance time was recorded using two sets of timing gates 
(TC-Timing System, Brower Timings, Utah, USA). Participants performed four consecutive trials in one direction before performing four trials in the other direction; the order in which directions were tested was randomized and counterbalanced. Participants' fastest trial in each direction was subsequently analysed. Overall CODS performance was the sum of participants' fastest trials in the clockwise and counterclockwise directions. Trials were separated by a recovery duration of 60 seconds.

To obtain ground reaction force data during the CODS test, the first cut was performed with the push-off (outside) foot contacting entirely within the force plate. Trials were excluded if the participant landed outside the confines of the force plate, this was retrospectively checked using video analysis. All of the participants' fastest trials met these criteria.

\section{RELIABILITY}

Reliability values for the CODS test utilized in the current study have been reported in a previous investigation within the same participant population (20); the inter-session coefficient of variation (three sessions) for fastest overall CODS test time was $1.1 \%$ (SEM: $0.04 \mathrm{~s}$; ICC: 0.97 ). The inter-session reliability for vertical stiffness obtained from unilateral drop jumping has also been established in the same population. The coefficient of variation (three sessions) for vertical stiffness in the stiff limb was $6.7 \%$ (SEM: $10.6 \mathrm{~N} \cdot \mathrm{m}^{-1} \cdot \mathrm{kg}^{-1}$; ICC: 0.98 ) and in the compliant limb was $7.6 \%$ (SEM: N.m ${ }^{-1} \cdot \mathrm{kg}^{-}$ 1; ICC: 0.96).

\section{STATISTICAL ANALYSIS}

Shapiro-Wilks tests were performed to assess for normality; all variables were considered to be normally distributed given an alpha level of $P>0.05$. A 3 (condition) 
$\times 2$ (pre- to post-intervention) repeated measures analysis of variance (ANOVA) was performed to analyse for the effect of the interventions and subsequent interactions. An additional repeated measures ANOVA was performed for post-intervention values alone, to analyse for differences between the interventions. The correlation between post-intervention vertical stiffness and overall CODS time was examined using Pearson's $r$. Pair-wise effect sizes (d) (8) were calculated and interpreted using the thresholds defined by Hopkins (13) where: $<0.20$ = trivial, 0.20-0.59= small, 0.60-1.19 $=$ moderate, $1.20-1.99=$ large , and $\geq 2=$ very large. Statistical significance for all analyses was set at an alpha level of $P \leq 0.05$ and all statistical procedures were conducted using the Statistical Package for the Social Sciences for Windows (v21.0; SPSS Inc., Chicago, USA). 


\section{RESULTS}

CHANGE OF DIRECTION SPEED

${ }^{* * *}$ Figure 4 Near Here ${ }^{* * *}$

CODS performances were significantly different between conditions $\left(F_{(2,18)}=7.14 ; P\right.$ $=0.005)$ (Figure 4). Pair-wise comparisons showed that performances in UNILATERAL were $1.7 \%$ faster than CONTROL $(P=0.011 ; d=-1.08)$, but not BILATERAL ( $1.0 \%$ faster; $P=0.14 ; d=-0.59)$; these effect sizes were both 'moderate'. BILATERAL performances were not different from CONTROL $(0.8 \%$ faster; $P=0.41$; $d=-0.48)$ although the effect size was also moderate. CODS performance time was significantly correlated to post-intervention vertical stiffness $(R=-0.31 ; P=0.046)$.

\section{${ }^{* * *}$ Figure 5 Near Here ${ }^{* * *}$}

There was evidence of some inter-individual variability in response to the interventions (Figure 5). Seven participants recorded their quickest CODS test performance following UNILATERAL, two following BILATERAL and one following CONTROL.

\section{*** Table 2 Near Here ${ }^{* * *}$}

There was a main effect of intervention on CODS performance time for participants' faster $\left(F_{(2,18)}=3.56 ; P=0.050\right)$ and slower $\left(F_{(2,18)}=6.70 ; P=0.007\right)$ limbs. Pair-wise comparisons were not significant for the faster limb, although the faster performances following UNILATERAL were associated with moderate effect sizes versus CONTROL $(P=0.079 ; d=-1.12)$ and BILATERAL $(P=0.37 ; d=-0.69)$ (Table 2). Performances for the slower limb were significantly faster following UNILATERAL than following CONTROL $(P=0.017 ; d=-0.86)$. Differences in ground contact times were not 
observed for the fast $\left(F_{(2,18)}=0.75 ; P=0.49\right)$ or slow $\left(F_{(2,18)}=1.46 ; P=0.26\right)$ limbs (Table 2).

STIFFNESS

*** Table 3 Near Here ***

There was a main effect of the intervention, such that there was a significant increase in vertical $\left(F_{(1,9)}=6.53 ; P=0.031\right)$ and ankle $\left(F_{(1,9)}=6.38 ; P=0.032\right)$ stiffness, but not knee $\left(F_{(1,9)}=2.80 ; P=0.13\right)$ stiffness, from pre- to post-intervention (Table 3). There was no significant interaction effect between time (pre- to post-intervention) and intervention for vertical $\left(F_{(2,18)}=2.58 ; P=0.10\right)$ and ankle $\left(F_{(2,18)}=0.39 ; P=0.68\right)$ stiffness, but there was a significant time by intervention interaction effect for knee stiffness $(F(2,18)=5.38 ; P=0.015)$ indicating that the change in knee stiffness was not uniform across all three conditions (Table 3).

Post-intervention vertical stiffness was significantly different between conditions $\left(F_{(2,18)}\right.$ $=5.16 ; P=0.017)$ (Table 3). Vertical stiffness was greater following BILATERAL (11\%; $P=0.019 ; d=0.31)$ and UNILATERAL $(14 \% ; P=0.049 ; d=0.39)$ versus CONTROL; there was no difference between BILATERAL and UNILATERAL $(-2.6 \% ; P=0.94 ; d$ $=-0.08)$. Post-intervention ankle $\left(F_{(2,18)}=0.41 ; P=0.67\right)$ and knee $\left(F_{(2,18)}=3.04 ; P=\right.$ 0.073) stiffness were not significantly different between conditions. A small effect size suggested greater ankle stiffness $(6.1 \% ; d=0.26)$ and knee stiffness $(15.7 \% ; d=0.58)$ following UNILATERAL versus CONTROL. A moderate effect size suggested greater knee stiffness $(16.6 \% ; d=0.61)$ following BILATERAL versus CONTROL. 


\section{DISCUSSION}

The aim of the current study was to establish if acute exercise interventions designed to augment vertical stiffness influenced CODS in a population of healthy males. It was hypothesized that both the bilateral and unilateral preparation strategies would significantly improve CODS test performance versus a control strategy. Effect size analysis reported that both BILATERAL and UNILATERAL improved CODS performance versus CONTROL. However, a significant improvement was only observed following UNILATERAL. As such, the experimental hypothesis cannot be fully accepted. In comparison to CONTROL, vertical stiffness was greater following both BILATERAL and UNILATERAL.

Following UNILATERAL, CODS test performance was $1.7 \%(d=1.08)$ quicker versus CONTROL and $1.0 \%(d=0.59)$ quicker versus BILATERAL. The effect of preconditioning interventions versus traditional dynamic warm-up practices on CODS has been evaluated previously in a selection of investigations. Reactive agility has been improved by $4.7 \%(d=1.2)$ following heavy leg press exercise (41) in amateur soccer players. Badminton specific CODS has been improved by $5.0 \%(d=0.83)$ following a weight vest loaded warm-up by Maloney, et al. (22) in professional badminton players. Nava (27) also noted significant improvements in T-test performance following weight vest loaded warm-up in collegiate athletes, although the presentation of their results did not permit the calculation of percentages and effect size. Whilst Sole, et al. (32) did not report significant improvements $(2.3 \% ; d=0.18 ; P=0.07)$ in $10 \mathrm{~m}$ shuttle test performance following heavy back squats in collegiate tennis and basketball players, $70 \%$ of participants recorded faster times than following a dynamic warm-up. The magnitude of CODS improvement observed in the current study was therefore less than has previously been reported in the literature, although differences in the CODS 
tests employed make it difficult to draw direct comparisons. Nonetheless, the low training status of participants in the current study in comparison to other investigation could explain why the magnitude of the performance enhancement was lower. Potentiation-based protocols have been established to carry a greater effect in welltrained cohorts $(21,34,37)$ and such improvements have also been observed with a shorter recovery duration $(21,34)$. The likely increase in type II fibre percentage (21) and ability to more quickly dissipate fatigue (34) associated with training status has been proposed to underpin this effect. The augmentation of pre-conditioning interventions that has been reported in trained individuals may also explain the variance in responses to the pre-conditioning intervention in the current study (demonstrated in Figure 5). However, as no training data was available for the participants sampled, a direct relationship cannot be determined. To establish whether the effect of this type of stiffness pre-conditioning intervention is greater in a trained population, this would need to be examined directly in future investigations.

The aforementioned studies which have reported CODS enhancements following preconditioning interventions have not attempted to examine the mechanisms by which these enhancements occur. It has previously been reported that vertical stiffness was the strongest predictor of CODS following regression analyses and that faster performers in the CODS test exhibited greater vertical stiffness (20). This supports the deterministic model of CODS proposed in Figure 1 and the hypothesis that increasing vertical stiffness will improve CODS. In comparison to CONTROL, post-intervention vertical stiffness was $11 \%(d=0.31)$ greater following BILATERAL and $14 \%(d=0.39)$ greater following UNILATERAL. The increases in stiffness observed in the current study are comparable to the respective increases of $11 \%(P<0.05)$ and $16 \%(d: 0.52$; $P=0.013$ ) observed by Comyns, et al. (9) and Moir, et al. (26) following heavy back 
squat interventions versus a post-warm-up baseline. However, Barnes, et al. (2) reported a greater increase of $20 \%(d: 0.76 ; 90 \% \mathrm{Cl}: 4 \%)$ following a weight vest loaded warm up versus a control warm-up, an intervention with greater similarity to the interventions performed in the current study. Comparisons with the Barnes et al. (2015) investigation are also more appropriate given that they are the only investigators, to the author's knowledge, to attempt to link performance enhancements to specific biomechanical variables. Barnes et al. (2015) reported an enhancement in performance (peak running speed) of $2.9 \%(90 \% \mathrm{Cl}: 0.8 \%)$, noting a 'very-high' correlation between the change in performance and the change in vertical stiffness ( $R$ $=0.88 ; 90 \% \mathrm{Cl}: 0.66-0.96)$. The current study reports a statistically significant relationship between increased stiffness and CODS, although this correlation $(R=$ 0.31 ) is weaker than that reported by Barnes et al. (2015).

Maloney, et al. (20) demonstrated that shorter ground contact times were associated with faster CODS performances, in agreement with previous investigations $(23,31)$. Increased stiffness would be expected to facilitate shorter ground contact times, as has been discussed previously, and could explain how greater stiffness may contribute to the enhancement of CODS. Whilst the shortest ground contact times were observed following UNILATERAL and the longest following CONTROL (Table 2), mirroring what was observed for CODS performance time, this relationship was not statistically significant and the effect sizes were small $(d<0.2)$. The likely reason for the lack of a relationship within the current study is that augmentations in ground contact time were small and inter-participant variation was large. For example, the difference in average ground contact time between UNILATERAL and CONTROL was $-1.6 \%$, and the standard deviation was $\sim 18 \%$ of the mean. 


\section{PRACTICAL APPLICATION}

The current study reports that a unilateral pre-conditioning intervention designed to augment vertical stiffness improved CODS performance in comparison to bilateral and control interventions. Also, improvements in CODS performance were related to greater post-intervention vertical stiffness. For healthy males preparing to engage in sports where CODS is an important factor, it is therefore recommended that preparation strategies include unilateral exercises designed to augment vertical stiffness. Examples utilized in this study included pogo hops and drop jumps. Whether this relationship is observed in well-trained athletic populations requires further investigation. 


\section{REFERENCES}

1. Arampatzis A, Schade F, Walsh M, and Brüggemann GP. Influence of leg stiffness and its effect on myodynamic jumping performance. Journal of Electromyography and Kinesiology 11: 355-364, 2001.

2. Barnes KR, Hopkins WG, McGuigan MP, and Kilding AE. Warm-up with a weighted vest improves running performance via leg stiffness and running economy. Journal of Science and Medicine in Sport 18: 103-108, 2015.

3. Bergmann J, Kramer A, and Gruber M. Repetitive hops induce postactivation potentiation in Triceps Surae as well as an increase in the jump height of subsequent maximal drop jumps. PloS one 8: e77705, 2013.

4. Bret C, Rahmani A, Dufour AB, Messonnier L, and Lacour JR. Leg strength and stiffness as ability factors in $100 \mathrm{~m}$ sprint running. Journal of Sports Medicine and Physical Fitness 42: 274-281, 2002.

5. Butler RJ, Crowell III HP, and Davis IM. Lower extremity stiffness: implications for performance and injury. Clinical Biomechanics 18: 511-517, 2003.

6. Byrne PJ, Kenny J, and O' Rourke B. Acute potentiating effect of depth jumps on sprint performance. Journal of Strength and Conditioning Research 28: 610-615, 2014.

7. Chen ZR, Wang YH, Peng HT, Yu CF, and Wang MH. The acute effect of drop jump protocols with different volumes and recovery time on countermovement jump performance. Journal of Strength and Conditioning Research 27: 154-158, 2013.

8. Cohen J. Statistical power analysis for the behavioral sciences. New Jersey: Lawrence Erlbaum, 1998.

9. Comyns TM, Harrison AJ, Hennessy L, and Jensen RL. Identifying the optimal resistive load for complex training in male rugby players. Sports Biomechanics 6: 59-70, 2007.

10. Delaney JA, Scott TJ, Ballard DA, Duthie GM, Hickmans JA, Lockie RG, and Dascombe BJ. Contributing factors to change-of-direction ability in professional rugby league players. Journal of Strength and Conditioning Research, 2015.

11. Dempster WT. Space requirements of the seated operator. Ohio: WrightPatterson Air Force Base, 1955.

12. Farley CT, Houdijk HHP, Van Strien C, and Louie M. Mechanism of leg stiffness adjustment for hopping on surfaces of different stiffnesses. Journal of Applied Physiology 85: 1044-1055, 1998.

13. Hopkins WG. A spreadsheet for analysis of straightforward controlled trials, in: Sportscience. 2003.

14. Keogh JWL, Weber CL, and Dalton CT. Evaluation of anthropometric, physiological, and skill related tests for talent identification in female field hockey. Canadian Journal of Applied Physiology 28: 397-409, 2003.

15. Kuitunen S, Ogiso K, and Komi PV. Leg and joint stiffness in human hopping. Scandinavian Journal of Medicine and Science in Sports 21: e157-e167, 2011.

16. Kümmel J, Bergmann J, Prieske O, Kramer A, Granacher U, and Gruber M. Effects of conditioning hops on drop jump and sprint performance: a randomized crossover pilot study in elite athletes. BMC Sports Science, Medicine and Rehabilitation 8: 1-8, 2016. 
17. Latash ML and Zatsiorsky VM. Joint stiffness: Myth or reality? Human Movement Science 12: 653-692, 1993.

18. Lima JCB, Marin DP, Barquilha G, Da Silva LO, Puggina EF, Pithon-Curi TC, and Hirabara SM. Acute effects of drop jump potentiation protocol on sprint and countermovement vertical jump performance. Human Movement 12: 324330, 2011.

19. Maloney SJ, Richards J, Nixon DGD, Harvey LJ, and Fletcher IM. Vertical stiffness asymmetries during drop jumping are related to ankle stiffness asymmetries. Scandinavian Journal of Medicine and Science in Sports, 2016.

20. Maloney SJ, Richards J, Nixon DGD, Harvey LJ, and Fletcher IM. Do stiffness and asymmetries predict change of direction performance? Journal of Sports Sciences 35: 547-556, 2017.

21. Maloney SJ, Turner AN, and Fletcher IM. Ballistic exercise as a pre-activation stimulus: a review of the literature and practical applications. Sports Medicine 44: 1347-1359, 2014.

22. Maloney SJ, Turner AN, and Miller S. Acute effects of a loaded warm-up protocol on change of direction speed in professional badminton players. Journal of Applied Biomechanics 30: 637-642, 2014.

23. Marshall BM, Franklyn-Miller AD, King EA, Moran KA, Strike SC, and Falvey ÉC. Biomechanical factors associated with time to complete a change of direction cutting maneuver. Journal of Strength and Conditioning Research 28: 2845-2851, 2014.

24. McGowan CJ, Pyne DB, Thompsen KG, and Rattray B. Warm-up strategies for sport and exercise: mechanisms and applications. Sports Medicine 45: 1523-1546, 2015.

25. Meir R, Newton R, Curtis E, Fardell M, and Butler B. Physical fitness qualities of professional rugby league football players: Determination of positional differences. Journal of Strength and Conditioning Research 15: 450-458, 2001.

26. Moir GL, Mergy D, Witmer CA, and Davis SE. The acute effects of manipulating volume and load of back squats on countermovement vertical jump performance. Journal of Strength and Conditioning Research 25: 14861491, 2011.

27. Nava KM. The acute effects of different warm-up techniques on power output, agility, and flexibility in athletes, in: Faculty of the College of Education. University of Texas at Brownsville, 2015.

28. Newton RU and Dugan E. Application of strength diagnosis. Strength \& Conditioning Journal 24: 50-59, 2002.

29. Read MM and Cisar C. The influence of varied rest interval lengths on depth jump performance. Journal of Strength and Conditioning Research 15: 279 283, 2001.

30. Reilly T, Williams AM, Nevill A, and Franks A. A multidisciplinary approach to talent identification in soccer. Journal of Sports Sciences 18: 695-702, 2000.

31. Sasaki S, Nagano Y, Kaneko S, Takakuni S, and Fukubayashi T. The relationship between performance and trunk movement during change of direction. Journal of Sports Science and Medicine 10: 112-118, 2011.

32. Sole CJ, Moir GL, Davis SE, and Witmer CA. Mechanical analysis of the acute effects of a heavy resistance exercise warm-up on agility performance in court-sport athletes. Journal of Human Kinetics 39: 147-156, 2013. 
33. Spiteri T, Newton RU, Binetti M, Hart NH, Sheppard JM, and Nimphius S. Mechanical determinants of faster change of direction and agility performance in female basketball athletes. Journal of Strength and Conditioning Research 29: 2205-2214, 2015.

34. Suchomel TJ, Lamont HS, and Moir GL. Understanding vertical jump potentiation: a deterministic model. Sports Medicine 46: 809-828, 2016.

35. Tobin DP and Delahunt E. The acute effect of a plyometric stimulus on jump performance in professional rugby players. Journal of Strength and Conditioning Research 28: 367-372, 2014.

36. Turner AP, Bellhouse S, Kilduff LP, and Russell M. Postactivation potentiation of sprint acceleration performance using plyometric exercise. Journal of Strength and Conditioning Research 29: 343-350, 2015.

37. Wilson JM, Duncan NM, Marin PJ, Brown LE, Loenneke JP, Wilson SMC, Jo $\mathrm{E}$, Lowery RP, and Ugrinowitsch C. Meta-analysis of postactivation potentiation and power: Effects of conditioning activity, volume, gender, rest periods, and training status. Journal of Strength and Conditioning Research 27: 854-859, 2013.

38. Winter DA. Kinetics: forces and moments of force, in: Biomechanics and Motor Control of Human Movement. New Jersey: John Wiley \& Sons, Inc., 2009, pp 107-138.

39. Young W, James $\mathrm{R}$, and Montgomery I. Is muscle power related to running speed with changes of direction? Journal of Sports Medicine and Physical Fitness 42: 282-288, 2002.

40. Young WB, Miller IR, and Talpey SW. Physical qualities predict change-ofdirection speed but not defensive agility in Australian rules football. Journal of Strength and Conditioning Research 29: 206-212, 2015.

41. Zois J, Bishop DJ, Ball K, and Aughey RJ. High-intensity warm-ups elicit superior improvements to a current soccer warm-up routine. Journal of Science and Medicine in Sport 14: 522-528, 2011. 


\section{FIGURE CAPTIONS:}

Figure 1 - The modified deterministic model of change of direction speed. Key: LPHC = lumbo-pelvic hip complex.

Figure 2 - The design of each experimental trial. Key: CODS = change of direction speed.

Figure 3 - An example of the experimental set-up for the change of direction speed test set up to examine right leg cutting performance; the set-up would be mirrored to examine left leg performance.

Figure 4 - Mean ( \pm standard deviation) change of direction speed test performances following each of the three interventions. ${ }^{*}$ indicates significantly faster than control $(P<0.05)$.

Figure 5 - Participants' change of direction speed test performances following each of the three interventions. 